\title{
Spontaneous Regression of a Histologically Proven Cutaneous Squamous Cell Carcinoma
}

\author{
Sweta Sengupta ${ }^{1}$, James Arocho ${ }^{2}$, T. Christopher Windham ${ }^{3}$ \\ ${ }^{1}$ Florida State University College of Medicine, Tallahassee, USA; ${ }^{2}$ Florida Hospital Memorial Medical Center, Memorial Hospital, \\ Daytona Beach, USA; ${ }^{3}$ Florida Hospital Memorial Medical Center, Comprehensive Cancer Center, Memorial Hospital, Daytona \\ Beach, USA. \\ Email:ss10d@med.fsu.edu
}

Received January $28^{\text {th }}, 2013$; revised February $28^{\text {th }}, 2013$; accepted March $7^{\text {th }}, 2013$

Copyright (c) 2013 Sweta Sengupta et al. This is an open access article distributed under the Creative Commons Attribution License, which permits unrestricted use, distribution, and reproduction in any medium, provided the original work is properly cited.

\begin{abstract}
Introduction: The rate of squamous cell carcinoma spontaneous regression (SR) remains unknown because incidences are rare and underreported. Case Report: We present a case of a 92-year-old Caucasian female who was found to have a $1 \mathrm{~cm}$ lesion on her nose. Pathologic evaluation supported the diagnosis of a moderately-differentiated squamous cell carcinoma. The patient refused treatment and returned with no clinical evidence of disease several months later. The lesion spontaneously regressed without excision. Discussion: Frequency of SR of malignancies has been estimated to occur 1 in 80,000 to 100,000 cases [1]. The biologic mechanisms of SR in malignancies remain unclear. Further investigations into the mechanisms of SR may identify potential treatment strategies for cancer.
\end{abstract}

Keywords: Squamous Cell Carcinoma; Spontaneous Remission

\section{Introduction}

Currently, the rate of squamous cell carcinoma spontaneous regression (SR) remains unknown. There has been much speculation as to mechanisms of SR. An immunologic mechanism has been proposed from clinical observations of an increased incidence of malignancies seen in immunosuppressed patients. Both in vivo and in vitro evidence supports an immunologic and hormonal mechanism in tumor regression. Alterations affecting apoptotic pathways of squamous cell carcinomas have been proposed as a mechanism for carcinogenesis and SR via $\mathrm{T}$ cell-mediated apoptosis. Both Cytotoxic $\mathrm{T}$ lymphocytes (CTLs) and Natural Killer cells (NK) can identify and induce apoptosis of cancer cells [2]. Existing immunological-based cancer therapies depend on CTLs. However, it has become increasingly clear that Helper T cells (HTLs) are critical for inducing tumor remission [3]. Combining cancer-targeting HTLS with chemotherapy has not been sufficiently investigated. Trophic effects from hormones on cancer cells have also been implicated in cases of SR [4]. Identifying and understanding mechanisms of SR may lead to innovative approaches in cancer therapeutics.

\section{Case Report}

A 92-year-old Caucasian female presented to her local dermatologist with a non-tender, one centimeter, centrally ulcerated lesion with raised margins arising on the nose (Figure 1(a)). She also reported tenderness regarding a separate 0.5 centimeter raised, well-circumscribed, non-ulcerated nodule on her right cheek. She had no lymphadenopathy or other significant findings on examination. A biopsy of her nose and cheek revealed a moderately differentiated squamous cell and well-differentiated squamous cell carcinoma, respectively. She refused our recommendation for resection of the lesion on the nose, insisting that only the tender squamous cell on the cheek be excised and failed to follow-up in our clinic. Eight months later, she returned and again requested removal of only her right cheek nodule. The lesion on her nose was no longer present (Figure 1(b)). She reported that the lesion on her nose was only treated with a daily application of rubbing alcohol by herself. She refused further head and neck evaluation but the nodule on her right cheek was removed; the right cheek nodule was discovered to be an invasive, well-differentiated squamous cell carcinoma (Figure 2). She has remained disease free since that incident. 


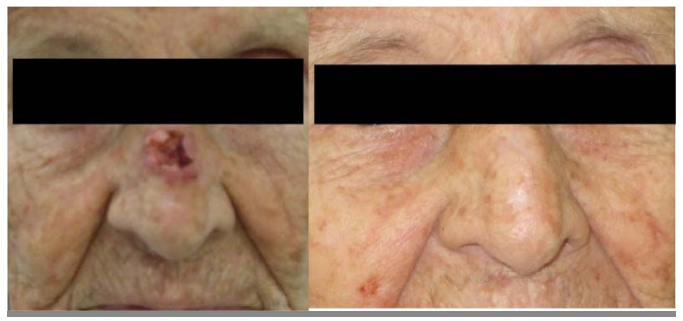

(a)

(b)

Figure 1. (a) One centimeter nasal centrally-ulcerated squamous cell cancer; (b) Eight-month follow-up of lesion.

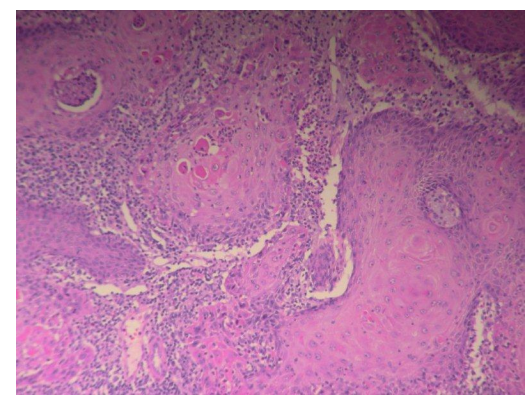

Figure 2. Invasive, well-differentiated squamous cell carcinoma.

\section{Discussion}

Cases of spontaneous remissions are rare and as a result, the rate among the general population remains unknown. "Spontaneous remission" can be defined as an incomplete or complete regression of a malignant growth in the absence of treatment or presence of treatment known to have no effect on cancer progression [5]. While SR has been more commonly reported in renal cell carcinoma, choriocarcinoma, retinoblastoma, and neuroblastoma [6], spontaneous regression of squamous cell carcinoma has long been a controversial phenomenon. Both keratoacanthoma, which is a benign neoplasm that commonly spontaneously regresses, and squamous cell carcinoma and share similar features [7].

The clinical presentation and histopathologic findings supported the diagnosis of squamous cell carcinoma in our patient. Our sample demonstrated numerous mitosis, marked pleomorphism, and moderate differentiation, favoring squamous cell carcinoma over keratoacanthoma. Keratoacanthomas represent a well-differentiated variant.

Cells are equipped to handle DNA damage and decrease chance of cancer progression via photoreactivation, base excision, mismatch, double-stranded break, and nucleotide excision repair [8]. However, the exact mechanism causing spontaneous regression remains elusive. Immunological basis for SR has been studied in melanomas. In one study, melanoma-related markers, particularly glycoproteins 75 and 110, GD2, and GD3 exhibited higher expression in regressing melanomas [9].
More evidence of the role of the immune system is demonstrated following organ transplantation. Immunosuppressed patients have a high incidence of squamous cell carcinoma development and lower rates of regression [10].

Mechanistically, SR can occur via T-cell mediated apoptosis. In vitro investigation of immunomodulation of neoplastic cells have been successful. Particularly, CTLs, leukocytes, monocytes, natural killer cells, lymphokineactivated killer cells, and rare plasma and mast cells have all been found to infiltrate tumors. Specifically, MHC class I Cytotoxic T lymphocytes have been found to have the greatest specificity for cancer cells [11]. CTLs facilitate remission via the release of granzyme $B$.

Squamous cell cancer is traditionally treated with surgical excision and intractable cases with surgery and platinum-based chemotherapy. Locally advanced, metastatic, and unresectable cases provide a greater challenge to treat. Targeting immunotherapies has demonstrated tremendous promise with treating intractable cases. Case reports of cetuximab, a monoclonal antibody targeting Epidermal Growth Factor Receptor (EGFR) has been associated with prolonged survival and is "well-tolerated” [12]. No large scale studies exist comparing cetuximab to chemotherapy treatment of non-melanoma skin cancers [12].

While significant evidence validates the immunological basis for cancer remission, regression may be nonimmunologic, as well. Elimination of carcinogens has been shown to result in cancer remission in certain cancers. However, this theory is incomplete. A prime example is smoking's influence on lung cancer. Smoking cessation does not induce cancer remission [5].

Hormones and trophic factors can have an effect on squamous cell cancer. In a Mancuso et al. study of two mice models, ovariectomized mice (with diminished estradiol and progesterone levels) have increased tumorogenesis and decreased tumor latency when compared to control mice. One theory is that estrogen is thought to bind with $\mathrm{ER} \beta$, which downregulates proto-oncogenic cyclin D1 transcription and ER $\alpha$ 's “proliferative action" on cancer cells [13]. Thereby, estrogen protects against aberrant cell growth in cells with high $\mathrm{ER} \beta /$ $\mathrm{ER} \alpha[13]$.

Other potential factor in inducing spontaneous remission, include cell re-differentiation via retinoic acids, growth factors, and other substances, natural killer cells, modulation of oncogene and tumor suppressors, anti-angiogenesis factors, apoptosis, and withdrawal of cellular stresses [5].

Further research into the underpinnings of spontaneous regression in malignancies may identify potential therapeutic treatment strategies for the prevention and treatment of malignancies. 


\section{Acknowledgements}

Luckey Dunn M. D., Dana V. Ferrence, R. N.

\section{REFERENCES}

[1] G. B. Challi and H. J. Stam, “The Spontaneous Regression of Cancer: A Review of Cases from 1900 to 1987," Acta Oncologica, Vol. 29, No. 5, 1990, pp. 545-550. doi:10.3109/02841869009090048

[2] F. H. Igney and P. H. Krammer, "Immune Escape of Tumors: Apoptosis Resistance and Tumor Counterattack," Journal of Leukocyte Biology, Vol. 71, No. 9, 2002, pp. 907-920.

[3] Z. C. Ding and G. Zhou, "Cytotoxic Chemotherapy and CD4+ Effector T Cells: An Emerging Alliance for Durable Anti-Tumor Effects," Clinical Development. Immunology, Vol. 2012, 2012, Article ID: 890178. doi:10.1155/2012/890178

[4] R. J. Papac, “Spontaneous Regression of Cancer,” Cancer Treatment Reviews, Vol. 22, No. 6, 1996, pp. 395-423. doi:10.1016/S0305-7372(96)90023-7

[5] W. Y. Chang, "Complete Spontaneous Regression of Cancer: Four Case Reports, Review of Literature, and Discussion of Possible Mechanisms Involve," Hawaii Medical Journal, Vol. 59, 2000, pp. 379-385.

[6] B. O’Regan and C. Hirschberg, "Spontaneous Remission. An Annotated Bibliography,” Institute of Noetic Sciences, Sausalito, 1996.

[7] J. Li, K. Wang, T. D. Jensen, S. T. Li, P. M. De Angelis, S. Kolvraa, C. Proby, O. Forslund, L. Bolund and O. P. Clausen, "Array Comparative Genomic Hybridization of
Keratoacanthomas and Squamous Cell Carcinomas: Different Patterns of Genetic Aberrations Suggest Two Distinct Entities," Journal of Investigative Dermatology, Vol. 132, No. 8, 2012. doi:10.1038/jid.2012.104

[8] S. D. Cline and P. C. Hanawalt, "Who's on First in the Cellular Response to DNA Damage?” Nature Reviews Molecular Biology, Vol. 4, No. 5, 2003, pp. 361-373. doi:10.1038/nrm1101

[9] F. J. Tefany, R. Barneston and C. Sta, "Immunocytochemical Analysis of the Cellular Infiltrate in Primary Regressing and Non-Regressing Malignant Melanoma," Journal of Investigative Dermatology, Vol. 97, No. 2, 1991, 197-202. doi:10.1111/1523-1747.ep12479662

[10] L. Jennings and C. D. Schmults, "Management of HighRisk Cutaneous Squamous Cell Carcinoma," Journal of Clinical Aesthetic Dermatology, Vol. 3, No. 4, 2010, pp. 39-48.

[11] S. Mocellin, E. Wang and F. Marincola, "Cytokines and Immune Response in Tumor Microenvironment,” Journal of Immunotherapy, Vol. 24, No. 5, 2001, pp. 392-407. doi:10.1097/00002371-200109000-00002

[12] S. J. Kalapurakai, J. Malone, K. Robbins, L. Beuscher, J. Godwin and K. Rao, "Cetuximab in Refractory Skin Cancer Treatment,” Journal of Cancer, Vol. 3, 2012, pp. 257261. doi:10.7150/jca.3491

[13] M. Mancuso, D. Gallo, S. Leonardi, M. Pierdomenico, E. Pasquali, I. De Stefano, S. Rebessi, M. Tanori, G. Scambia1, V. Di Majo, V. Covelli, S. Pazzaglia and A. Saran, "Modulation of Basal and Squamous Cell Carcinoma by Endogenous Estrogen in Mouse Models of Skin Cancer," Carcinogenesis, Vol. 30, No. 2, 2009, pp. 340-347. doi:10.1093/carcin/bgn243 